\title{
La praxis como fundamento de una educación para la salud alternativa: estudio de investigación-acción en el Programa de Crecimiento y Desarrollo en Medellín, Colombia
}

\author{
Fernando Peñaranda-Correa ${ }^{1}$ \\ Julio Nicolas Torres-Ospina² \\ Miriam Bastidas-Acevedo ${ }^{3}$ \\ Gloria Escobar-Paucar ${ }^{4}$ \\ Adriana Arango-Córdoba ${ }^{5}$ \\ Fráncy Nélly Pérez-Becerra ${ }^{6}$
}

PEÑARANDA-CORREA, F. et al. Praxis as a foundation for an alternative health education: an action research study in the Growth and Development Monitoring Program in Medellin, Colombia. Interface - Comunic., Saude, Educ., v.15, n.39, p.997-1008, out./dez. 2011.

The aim of this study was to reflect on Freire's Education Model in a health education environment in order to promote its theoretical development. An action research was developed, whose object was the Growth and Development Monitoring Program of children in Medellin, Colombia. The study involved the use of participant observation in the meetings of this program, and analysis of the information focusing on a continuous reflective process. Educators-researchers analyzed their own pedagogical practices, aiming to transform health education into a more dialogic and productive practice. Findings showed difficulties to change traditional educational practices in the scope of health services, but from a praxis perspective, reflection was found to be an important process in transforming practice. The emergence of the student as a subject with specific experiences and conditions helped the educatorsresearches to understand the meaning of a human-based education. The need to consider caregivers as central subjects and child rearing as a central topic in health programs was highlighted.

Keywords: Health education. Child rearing. Growth and development.
La propuesta fue reflexionar sobre la educación problematizadora en un ambiente de educación para la salud y contribuir a su desarrollo teórico, en un proceso de investigación-acción, teniendo como objeto el programa de crecimiento y desarrollo en Medellín. El estudio incluyó el uso de la observación participante del programa de desarrollo de los niños y análisis de la información centrada en un proceso reflexivo permanente. Los educadores-investigadores analizaron sus prácticas pedagógicas, intentando transformar la educación en salud en una práctica más dialógica y productiva. Se encontraron dificultades para cambiar la educación tradicional en el ámbito de los servicios de salud. Se evidenció la importancia de la reflexión en la transformación de la práctica. El educando como sujeto, con experiencias y condiciones particulares contribuyó a la comprensión de una educación centrada en el ser humano. Se resaltó la necesidad de considerar los adultos como agentes centrales y la educación infantil como eje del programa.

Palabras clave: Educación en salud. Educación del niño. Crecimiento y desarrollo.

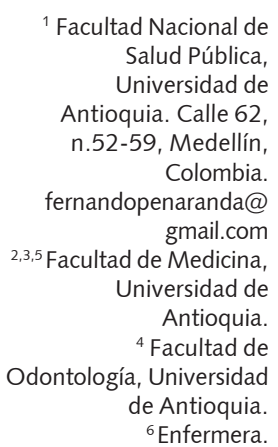




\section{Introducción}

La educación para la salud ha cumplido un papel importante en la salud pública, reconocido en escenarios como las conferencias de Alma Ata (OPS, 1978) y Ottawa (OMS, 1986). Aun así, existe controversia sobre sus orientaciones pedagógicas y sus logros (OMS/OPS, 2006; Nutbeam, 2006; Gazinelli, 2005; Morrel et al., 2000).

La pedagogía tradicional y la pedagogía conductista, que algunos agrupan como pedagogía "instruccional" (González, 1999), configuran la base pedagógica predominante de la educación para la salud, situación que puede explicarse, al menos, por dos razones. La primera la constituye el conservadurismo del modelo educativo tradicional, bajo el cual los profesionales de la salud enseñan al mínimo coste, esto es, con base en su experiencia en el sistema formal de educación (Bourdieu, Passeron, 1998). La segunda se refiere a la estrecha relación de la pedagogía conductista con el modelo biomédico, en sus tres características básicas: una orientación normalizadora, su fundamento en la ciencia positivista, (Robinson, Hill, 1999; Achard, 1980; Foucault, 1977) y finalmente su carácter medicalizador (Foucault, 1977).

Implementar perspectivas alternativas de educación para la salud que confronten el modelo biomédico y la forma convencional de entender la salud, centrada en la enfermedad, ha sido un asunto muy complejo, y generalmente los intentos se quedan en el discurso o en asuntos formales (PeñarandaCorrea et al., 2006; Gazinelli, 2005; Greene, Simons-Morton, 1988). En relación con los logros de la educación para la salud, su controversia no es menos aguda. A propósito, la OMS/OPS expresan:

A pesar del desarrollo de nuevas estrategias de educación en salud el avance en este tema no es evidente [...] El modelo medicalista continúa influenciando en forma desmedida un enfoque por enfermedad y riesgo, menospreciando la influencia de las condiciones y los determinantes sociales. (OMS/OPS, 2006, p.8)

En Colombia surgió desde la década del setenta el Programa de Crecimiento y Desarrollo como un componente de la atención materno-infantil. En el marco de la reestructuración del Sistema de Salud, se denominó desde el año 2000 como "Norma técnica para la detección temprana de las alteraciones del crecimiento y desarrollo en el menor de diez años" (Colombia, 2000), incluido en el Plan Obligatorio de Salud.

La historia del programa en la ciudad de Medellín provee una interesante experiencia con respecto al intento por promover una perspectiva alternativa al modelo preventivista. Se fomentó un modelo de atención grupal con el propósito de fortalecer la dimensión educativa del programa y socializar las experiencias de las madres respecto a la crianza de sus hijos. Como fruto de esta experiencia y bajo la influencia de la Carta de Ottawa, a principios de los noventa se realizó una propuesta desde la concepción de promoción de la salud, con la cual se buscó trascender la orientación morbicéntrica que prevalecía hasta el momento. Se cambió entonces el nombre de "Crecimiento y Desarrollo" por el de "Salud Integral para la Infancia" (SIPI), en un intento por desarrollar un programa desde la salud y no desde la enfermedad, y cuya dimensión educativa estaba fundada en el diálogo de saberes.

Desde finales de la década pasada, nuestro equipo de investigación ha realizado varios estudios para comprender la dimensión educativa del programa. Se encontró que la propuesta SIPI había logrado cambios importantes, relacionados, en particular, con la incorporación de profesionales de diferentes áreas de la salud y de las ciencias sociales al programa, lo cual mejoró el diagnóstico y la atención de las alternaciones del crecimiento y desarrollo de los niños, así como la información suministrada. Sin embargo, se evidenció que aun educadores conocedores y comprometidos con la propuesta de diálogo de saberes terminaban realizando prácticas pedagógicas centradas en modelos informativos y persuasivo-motivacionales que correspondían al modelo que se pretendía transformar (Peñaranda-Correa et al., 2006).

Los hallazgos revelaron que se produce una resignificación de las propuestas alternativas, dentro de las concepciones hegemónicas, que termina por neutralizar su poder transformador y que mantiene el statu quo (Peñaranda-Correa et al., 2006). De acuerdo con Tones (2002), persisten en la educación para 
la salud la imposición unilateral de contenidos y significados por parte de los profesionales de la salud y la adopción de posiciones poco dialógicas y poco democráticas. Lo anterior, contrapuesto a las expectativas de los adultos significativos para el logro de una crianza más segura, satisfactoria y productiva en aras de su propio bienestar y el del niño, evidenció que en el programa opera un encuentro entre racionalidades diferentes: los cuidadores, desde el saber cotidiano, y los profesionales de la salud, desde la perspectiva biomédica (Bastidas-Acevedo et al., 2007).

Pero la transformación del modelo pedagógico implica además otro reto: llevar a la práctica la comprensión teórica desarrollada. Como lo sostienen varios autores, no se da una directa aplicación de la teoría a la práctica (Gazzineli, 2005; Greene, Simons-Morton, 1988) y es necesario transitar por el camino de una investigación más ligada a la acción, en el marco de la espiral de la praxis: conceptualización-práctica-reflexión (Cendales, 2001; Fals Borda, 2001; Bosco Pinto, 1987). Con el propósito de avanzar en la construcción de una perspectiva educativa para el Programa de Crecimiento y Desarrollo, basada en el diálogo de saberes, y de aportar al desarrollo teórico en educación para la salud que promueva alternativas al modelo instruccional fundado en el modelo biomédico, el equipo de investigación se embarcó en un proyecto de investigación-acción, cuyos resultados se presenta a continuación.

\section{Metodología}

En tanto la intención era profundizar en un proceso reflexivo sobre la práctica educativa en el ámbito de la salud, se retomó a Stenhouse (2004) y su propuesta de una educación basada en la investigación, en la cual el educador se convierte en un investigador de su propia práctica educativa. Para este autor, la investigación en el campo de la educación debe centrarse en la praxis, de tal forma que, partiendo de la práctica educativa y por medio de la reflexión, el educador pueda acceder a una perspectiva teórica enriquecida para regresar a la práctica y resolver problemas concretos. Stenhouse entendía la investigación como un instrumento para desarrollar conciencia crítica sobre la educación desde una perspectiva emancipadora. En el mismo sentido, Freire $(2004,1975)$ se pronuncia sobre la importancia de sustentar la educación en la praxis, y Hatton y Smith (1995) destacan la trascendencia de los procesos reflexivos para la formación de los maestros. En concordancia con estos planteamientos, se llevó a cabo una investigación-acción cuyas características centrales, según Reason y Bradbury (2001), son: la producción de conocimiento útil para los involucrados, el logro de nuevas formas de comprender la realidad, la participación de todos los implicados y el desarrollo de un proceso emergente y evolutivo tan importante como los resultados mismos.

Desde este referente metodológico y epistemológico, se decidió realizar la investigación en el marco del Programa de Crecimiento y Desarrollo de la institución de salud adscrita a la Universidad de Antioquia, en Medellín, Colombia. Al Programa asisten, con sus hijos, estudiantes, profesores y empleados no docentes de la institución, y en él participan regularmente tres de los investigadores, en calidad de profesores de pediatría. El equipo investigador tendría entonces un doble papel: el de educadores como integrantes del equipo de salud y el de investigadores de su propia práctica educativa.

Entre febrero y agosto del 2007, se avanzó en la etapa de preparación interna del equipo a través de la reflexión teórica sobre diálogo de saberes. Se partió de una revisión de los principales postulados teóricos de Paulo Freire, complementada con otros autores de la corriente de educación popular. Para Bosco Pinto (1987, p.55-6): "la teorización no debe quedarse simplemente como un trabajo verbal o mental del equipo. Es indispensable hacer un esfuerzo para escribirla con el propósito de que objetivándose, se transforme en un instrumento de trabajo a ser utilizado en las fases ulteriores de la metodología".

En este sentido, la preparación teórica originó un artículo que presentó las reflexiones de una educación para la salud pensada desde el diálogo de saberes (Bastidas et al., 2009), paso previo a los demás momentos de la investigación.

El proyecto fue aprobado por el Comité de Ética de Investigación de la Facultad Nacional de Salud Pública. Los adultos significativos participaron de manera voluntaria, después de explicarles los objetivos 
de la investigación, de garantizarles la confidencialidad y de plantearles la posibilidad de retirarse en cualquier momento, además de que podían continuar en el Programa regular de la institución.

Entre agosto del 2007 y octubre del 2008, se llevó a cabo el desarrollo de la propuesta educativa, con un grupo de 11 niños y sus cuidadores adultos significativos (11 madres, 8 padres, 5 abuelas y un abuelo) asistentes al programa, quienes participaron como educandos. Se desarrollaron diez sesiones, desde el ingreso de los niños al programa cuando tenían un mes de vida hasta que cumplieron los catorce meses. Algunas sesiones se desarrollaron con los adultos significativos y sus hijos, bajo el formato regular del programa, que incluye actividades educativas y el monitoreo del crecimiento y desarrollo. Otras, intercaladas con las anteriores, se realizaron únicamente con la presencia de los adultos significativos para reflexionar sobre la crianza y el proceso educativo vivido. La asistencia a las sesiones fue, en general buena. Hacia el final de la investigación dejaron de asistir tres niños con sus respectivos acompañantes.

Las actividades educativas de las sesiones con niños y adultos significativos se iniciaban con un saludo general de los asistentes. Posteriormente cada uno de los adultos significativos compartía su experiencia en la crianza y sus inquietudes respecto a ésta. Seguidamente se generaba una discusión donde participaban tanto los adultos significativos como los educadores - investigadores para problematizar y analizar los asuntos expuestos. Al finalizar la sesión se hacía un cierre para evaluar los principales logros, avances, inquietudes y dificultades en torno a la crianza.

En las sesiones con adultos significativos solos, una vez hecho el saludo de costumbre y haber compartido las experiencias vividas e inquietudes respecto a la crianza, se pasaba a un momento de reflexión y análisis sobre la crianza misma y el proceso educativo vivido en el programa.

A medida que avanzaron las sesiones los adultos significativos fueron adquiriendo más confianza y se hicieron más expresivos para compartir sus experiencias y el contexto en el que estaban viviendo la crianza. Cada vez profundizaron más en sus reflexiones sobre asuntos íntimos y de gran impacto emocional.

La recolección y análisis de la información se realizaron de manera simultánea y su desarrollo se dio en dos ámbitos: las sesiones con los adultos significativos, registradas en diarios de campo, y las reuniones del equipo investigador, plasmadas en actas. El análisis se centró en un proceso de reflexión sistemática del equipo investigador, dado que la particularidad del proyecto requería cambios inmediatos y continuos en el proceso educativo. La investigación se fundamentó en la praxis como un asunto dialéctico de acción-reflexión y teorización, que permitió reorientar permanentemente el desarrollo investigativo hacia los aspectos más significativos (Lincoln, Guba, 2000).

\section{Resultados}

Como producto de la praxis, se generó un proceso gradual de transformación de las prácticas educativas en el marco del programa, a partir de la propia transformación de los investigadoreseducadores, de la transformación de los educandos y de la transformación, tanto de los ambientes de aprendizaje como de las relaciones construidas en el seno de estos. Si bien corresponden a un proceso integrado, para fines expositivos los resultados se presentarán a manera de tres grandes categorías: el surgimiento del sujeto, las prácticas pedagógicas y, finalmente, la construcción de una identidad grupal y la transformación de los educandos. Asimismo, el texto toma la forma de relato, en tanto los hallazgos se fundamentaron en la praxis de los investigadores en el marco de un proceso de sistematización.

\section{El surgimiento del sujeto}

Durante el transcurso de las sesiones, pasamos de un conocimiento superficial de los educandos hacia una comprensión más profunda de cada uno de ellos y sus situaciones particulares. Así, los adultos significativos-educandos surgieron como sujetos: ya no era un grupo homogéneo, sino un grupo de personas, cada una con una historia y unas condiciones singulares, vividas por individuos concretos en un contexto particular. Con ello, fuimos comprendiendo de una manera más amplia las condiciones 
sociales, culturales y humanas en las cuales estos adultos llevaban a cabo la crianza de sus hijos. Paulatinamente, hicimos un tránsito hacia el reconocimiento de sus expectativas, alegrías, tristezas, sueños y frustraciones en relación con la crianza, con su rol en ella y con las transformaciones que operaban en los ámbitos personal, familiar, laboral o escolar, según el caso. En cada uno de aquellos adultos había un drama complejo, lleno de conflictos, para los cuales se generaban, de manera continua, equilibrios inestables y cambiantes.

Este hallazgo mostró las limitaciones de una educación basada en recomendaciones generales, comunes para todos, que busca la homogenización. Se encontró que nuestros discursos como profesionales-educadores tenían distintos sentidos para los educandos, quienes los resignificaban de acuerdo con sus condiciones y características personales y socioeconómicas. Asimismo, reconocimos que cualquier indicación normativa podría tener efectos muy diversos, algunos positivos y otros negativos, debido a la complejidad de los equilibrios que los adultos significativos debían negociar de manera permanente en su cotidianidad. Con ello, el equipo empezó a mirar con prudencia la tendencia a reaccionar mediante una "intervención" (prescripión) en la vida de los demás.

\section{Las prácticas pedagógicas}

Tres sucesos centrales fueron decisivos en la transformación de nuestras prácticas pedagógicas: una crisis respecto a nuestro papel como educadores, la consolidación progresiva de los propósitos que guiaron la experiencia educativa y la transformación de los contenidos en "temas generadores" (Freire, 1975, p.112).

En las primeras sesiones, nos vimos abocados a una crisis sobre nuestro papel como educadores. Nos dimos cuenta de lo difícil que era para nosotros llevar a la práctica las concepciones de una educación alternativa. Nos costaba trabajo escuchar a los educandos: tendíamos a interpretar sus inquietudes desde las categorías biomédicas y la respuesta a las mismas se daba sin indagar por el sentido que éstas tenían para los educandos. Reconocer lo difícil que era superar el modelo "instruccional" tan cuestionado en trabajos anteriores produjo una crisis de identidad en el equipo y generó inseguridad sobre la relación con los conocimientos disciplinares y con el conocimiento de los educandos. Esta falta de coherencia entre los ideales y las acciones nos generó frustración.

Sin embargo, la crisis se empezó a resolver en la medida en que, sin desechar nuestros conocimientos disciplinares, estos se fueron relativizando para acceder a posiciones más plurales. De otro lado, la lectura de Freire (2004) nos mostró que la coherencia es una meta por alcanzar, y ello requiere un profundo y continuado esfuerzo de reflexión y autocrítica. Hacia el final de la octava sesión, se logró mayor claridad sobre los propósitos que debían guiar una educación basada en el diálogo de saberes para el programa, consignados así:

- promover el desarrollo humano de los adultos significativos y su seguridad para realizar una crianza más productiva y satisfactoria para ellos y para los niños y niñas;

- generar una experiencia educativa que facilite mayor comprensión de la crianza que los adultos significativos se encuentran realizando, de tal manera que se convierta en un espacio para que ellos puedan comprenderse mejor como personas y en su función de crianza, y apoyar así la construcción de su identidad;

- generar un ambiente educativo propicio para apoyar a los adultos significativos en sus necesidades respecto de la crianza, cualificando nuestra capacidad para escucharlos y aprender de ellos.

Estos propósitos buscaban consolidar una propuesta centrada en el sujeto, que facilitara a los educandos la construcción de principios generales sobre la crianza, orientados desde sus particularidades y en correspondencia con los significados que estos tenían para ellos. Habíamos logrado trascender la preocupación por verificar el cumplimiento de comportamientos preestablecidos por el conocimiento biomédico. La preocupación se centraba más bien en comprender como estos principios, construidos por cada uno de ellos, les eran útiles para sus vidas, para comprender mejor la crianza y comprenderse mejor ellos mismos.

Siguiendo a Freire y su idea del diálogo como un acto de "fe en los hombres" (Freire, 1975, p.104), a medida que ganábamos más confianza en los adultos como educandos y en nosotros como 
educadores, se pasó gradualmente de sesiones fuertemente estructuradas, con un derrotero de contenidos preestablecidos, a sesiones más flexibles. De esta manera, las sesiones, más que como instancia para informar, se concibieron como escenario para tramitar las inquietudes, las necesidades y los intereses de los educandos. Sin embargo, este cambio estaba cruzado por un conflicto alrededor de los intereses dispares entre educandos y educadores. Un formato abierto y más libre siempre planteaba la inquietud de dejar por fuera de la discusión conocimientos importantes para los adultos significativos en su función de crianza y para la salud de los niños.

Reconocimos la imposibilidad de desarrollar todos los temas que a nosotros se nos ocurrían como necesarios o, por lo menos, la imposibilidad de trabajarlos de forma productiva para ellos y nosotros. El proceso educativo no debería estar centrado entonces en la enseñanza, sino en el aprendizaje: los adultos aprenderían más sobre aquello que necesitaban, y el desarrollo de un conocimiento productivo estaría relacionado con una vinculación "vital-afectiva" por parte de los educandos.

Surgió la idea de trabajar con base en temas generadores, como lo propuso Freire, orientados a facilitar en los educandos un análisis más amplio y profundo de su realidad, que propiciara su propia transformación y la de sus situaciones concretas. Esperábamos llevar a cabo una secuencia de codificación y decodificación que permitiera analizar de manera conjunta con los adultos significativos sus propias situaciones de crianza, y compartir con ellos la construcción teórica que como profesionales nos había permitido llegar a una comprensión más profunda de la crianza. Pero la experiencia nos mostró que no se avanzaba hacia una visión de conjunto, como lo esperábamos, construyendo una explicación teórica ordenada y articulada con categorías bien definidas. Se avanzaba más bien hacia procesos reflexivos que permitían a los educandos construir verdades secuenciales y cambiantes, referidas a contextos particulares, y articuladas desde referentes distintos a la lógica con la cual los profesionales de la salud estamos acostumbrados a trabajar. Otras lógicas operaban en estos análisis: afectivas y culturales.

Con esta experiencia cambió la concepción de temas generadores y la forma de desarrollarlos en las sesiones. Se decidió entonces trabajar con base en las inquietudes y problemas propuestos por los educandos, en sus propios términos, al entender que la vinculación afectiva y la tramitación de las emociones eran centrales para generar un proceso reflexivo, que ahora se consideraba la base de nuestra práctica educativa. Ese proceso permitía a los educandos relacionar su acción con los conocimientos $y$, en este orden de ideas, tener en cuenta una teoría, pero no la teoría que nosotros habíamos construido, sino la que cada uno de ellos elaboraba para explicar su propia realidad y encontrar soluciones pertinentes a sus propias situaciones y características personales.

\section{Construcción de una identidad grupal y transformación de los educandos}

La construcción paulatina de una identidad de grupo con la cual educandos y educadores se sintieron tranquilos fue fundamental para el logro de prácticas pedagógicas más dialógicas y la construcción de relaciones más productivas. Así, en la medida en que se fue generando un espacio más abierto y de mayor confianza entre los participantes, los educandos asumieron un papel más activo y constructivo.

Los adultos significativos comenzaron a reconocer más ampliamente la importancia de sus propios conocimientos y a concebir el escenario educativo como una instancia de análisis y reflexión, donde cada uno compartía sus puntos de vista para construir conocimientos, ideas y referentes, con el fin de tomar sus propias decisiones. El haber compartido sus angustias, necesidades, problemas y situaciones particulares con otras personas que atravesaban por situaciones similares, en un ambiente comprensivo, permitió a los adultos significativos sentirse escuchados y no juzgados, con lo cual encontraron apoyo para construir sus identidades como madres o padres.

Este sentimiento grupal se vio reforzado en el transcurso del programa entre educandos que se fueron compenetrando y solidarizando entre sí. Con ello, se observó cómo en las sesiones buscaban espacios para compartir sus experiencias de crianza, sus preocupaciones y sus alegrías; se mostraban interesados en los problemas de los demás y querían aportar en búsqueda de soluciones. Este proceso de integración no trascendió el espacio del programa —una de nuestras expectativas iniciales—, pero fue un elemento clave en la construcción de relaciones y ambientes de aprendizaje más productivos. 


\section{Los resultados}

Con el correr del tiempo, los educandos modificaron algunas situaciones conflictivas que implicaba la crianza, pero también se encontraron con problemas que no pudieron cambiar de manera sustancial. Sin embargo, aunque algunas situaciones no se hubieran modificado drásticamente, sintieron que el hecho de "desahogarse" y compartir sentimientos y experiencias que nunca habían expresado a otros, así como conocer otras vivencias igualmente difíciles, les había servido para mitigar su angustia y frustración, pero también para aprender a mirar de modo diferente su realidad, en la medida en que la comprendían desde un contexto más amplio.

En las dos últimas sesiones se realizó una evaluación que buscaba promover la reflexión sobre la crianza que habían vivido durante los catorce meses transcurridos y el proceso educativo llevado a cabo en el programa. Los adultos manifestaron sentirse más competentes para la crianza, principalmente como producto de su interacción con los niños y con su entorno, pero también reconocieron el papel de apoyo del programa y el aprendizaje logrado en él. Como educandos, habían tomado sus propias determinaciones de manera tranquila, sin sentirse juzgados o intimidados en el programa: un logro relacionado con haber llevado a cabo un proceso de discusión y reflexión sobre sus vidas y sus prácticas de crianza que les permitió desarrollar una posición más crítica hacia estas.

Este análisis final nos sirvió para reconocer el valor de comprender las necesidades del educando y respetar su autonomía, permitiendo que tome decisiones acordes con su contexto. En estas condiciones, el proceso educativo es útil para ayudarles a resolver asuntos complejos y a fortalecer su criterio y seguridad en su función de crianza, de allí que la evaluación de un programa como el de crecimiento y desarrollo debería trascender la verificación de "logros" impuestos desde la perspectiva biomédica y darle una mayor importancia al desarrollo de las personas.

\section{Discusión}

La crisis respecto del papel del equipo como educadores mostró que la implementación de una propuesta de educación alternativa al modelo "instruccional" y al modelo biomédico implica ir más allá del planteamiento de Bourdieu, respecto a que el educador requiere conocer "los mecanismos más elementales de la transmisión cultural [...] para combatir lo que yo llamo efecto de destino, y la tendencia de los profesores a creer que ellos juzgan a la persona, cuando en vedad juzgan a individuos sociales" (Bourdieu, 1998, p.174). Se requiere también de un profundo proceso de reflexión crítica sobre la práctica pedagógica, un proceso penoso y difícil, en tanto exige una "vigilancia constante que tenemos que ejercer sobre nosotros mismos para evitar los simplismos, las facilidades y las incoherencias burdas" (Freire, 2004, p.49). Adecuando los términos de Bourdieu, no es fácil transformar el habitus "pedagógico" del educador, lo cual sustenta la pertinencia de la investigación-acción para el campo de la educación, como lo sostiene Stenhouse (2004).

De otro lado, la fuerza con la cual irrumpió el educando como sujeto en esta investigación hizo focalizar la mirada en el ser humano, no solo en relación con la dimensión pedagógica, sino también en cuanto a la concepción de salud. Se reconoció con ello una perspectiva autopoyética de la salud (Granda, 2008), con un ser humano que construye su salud en interacción con su medio ambiente; en este sentido, es posible afirmar que corresponde a una visión ontológica de la salud, en la cual el individuo "es" saludable, siéndolo a su manera. La salud tiene que ver con el desarrollo humano y el desarrollo de capacidades (Sen, 1996), lo que implica, a su vez, la construcción del mundo social con el que el sujeto interactúa (Freire, 2004) y, por ende, superar la dualidad individuo-ambiente (Granda, 2008; Carvalho, 2005).

A partir de los resultados, también se comprendió que la salud constituye un "estado de equilibrio [...]. Pero todo intento de compensar una perturbación mediante un contrapeso significa, a la vez, la amenaza de una nueva pérdida de equilibrio en el sentido contrario" (Gadamer, 1996, p.128-9). Reflexionar sobre los equilibrios inestables y continuados que los adultos significativos constantemente deben negociar en sus cotidianidades ponen de manifiesto la necesidad de rescatar la racionalidad prudente (phronesis) para el educador (Granda, 2008; Gadamer, 1996). 
Desde este punto de vista, parecen insuficientes las clasificaciones respecto de los modelos de educación para la salud que proponen Salleras et al. (2008), Serrano (1990), Greene y Simons-Morton (1988), Sharma y Romas (2007), así como las salidas para superar la inconmensurabilidad paradigmática de los referentes pedagógicos, por un lado ecléctica o, por otro, integracionista. Más bien habría que reconocer un mundo plural respecto de los modelos pedagógicos en la educación para la salud y plantear opciones a los modelos convencionales desde sólidas fundamentaciones epistemológicas y pedagógicas. Para ello, los insumos teóricos que aportan Freire y la educación popular latinoamericana constituyen valiosas contribuciones para avanzar hacia la construcción de propuestas alternativas.

Cuando se precisaron los propósitos de la educación que se estaba llevando a cabo se partió de un proceso centrado en el ser humano. Con ello se reconoció la pertinencia de considerar al ser humano como un ser consciente, crítico, autónomo y libre; un ser trascendente, transformador, que crea y recrea, que conoce y comprende y que está abierto a la realidad; un ser histórico, social y cultural, que no está solo y, por lo tanto, está en relación con el mundo y con otros (Freire, 2005, 2004, 1998, 1975).

El diálogo es, en esencia, un proceso comunicativo que se da en el marco de un contexto cultural (Freire, 2005). Siguiendo a Habermas (1987), se puede afirmar que el diálogo de saberes requiere una posición "fenomenológica", en la cual el mundo objetivo es una construcción intersubjetiva a través de la argumentación. Ello contrasta con la utilización no comunicativa de un saber proposicional, basado en un estado de cosas y en un mundo objetivo, ajeno al individuo, que constituye el ámbito de la ciencia. Esta perspectiva comunicativa implica reconocer la presencia del mundo de la vida como sustento de toda comprensión (Habermas, 1987) o, como lo plantea Bruner (1998), que la psicología popular o sentido común, aunque cambie, no se ve sustituida por paradigmas científicos. Lo anterior pone la educación para la salud en el terreno de la interpretación, es decir, de la subjetividad humana y, por tanto, en una perspectiva constructivista de la realidad.

En este contexto, es posible avanzar más allá de una educación centrada en la información y el desarrollo de unos contenidos preestablecidos por los agentes de salud. A pesar de las fuerzas que ejercen el modelo biomédico y los mecanismos de la transmisión cultural, cabría la posibilidad de encontrar vías alternas desde la "educación problematizadora" (Freire, 1975, p.132). Siguiendo a este mismo autor (1996, 1975), problematizar quiere decir analizar los fenómenos —en este caso, la crianza como experiencia humana - desde una perspectiva más amplia, identificando fuerzas, influencias y condicionantes, de tal manera que pueda superarse lo particular para acceder a una perspectiva general y poder así resignificar la realidad y la acción personal como punto de partida para fortalecer la capacidad de transformarlas. En este orden de ideas, desde una dimensión problematizadora, se entendió que al narrar sus experiencias, en el marco de un proceso de escucha y de reflexión, los educandos ampliaron la comprensión de su situación como personas y "criadores" en un contexto histórico, social, cultural y económico particular. Las narrativas, el proceso reflexivo y la construcción de principios generales como teorías explicativas y morales ligadas a la racionalidad cotidiana permitieron la transformación de ellos como personas e, incluso, facilitaron algunos cambios de sus situaciones sociales. En este sentido, con Dewey (Hatton, Smith, 1995), se reconoció en el pensamiento reflexivo un pensamiento orientado a la solución de problemas y hacia el desarrollo de una conciencia crítica (Freire, 1975). Así, se promueve en los educandos (y también en los educadores) la capacidad de revisarse a sí mismos y su situación en medio de un ambiente de discusión sobre sus condiciones como individuos localizados en un contexto social, económico y cultural. De esta manera, aunque no hubieran logrado hacer grandes cambios en las condiciones materiales de manera inmediata, los educandos se localizaron de manera diferente hacia las "situaciones límites" (Freire, 1975), con lo cual se perfila la posibilidad de transformaciones en el horizonte.

Pero en este caso, la educación problematizadora se planteó también en un ambiente de soporte y comprensión, dadas las situaciones conflictivas de los adultos significativos. Así, la identidad grupal y el desarrollo de la capacidad de escucha constituyen asuntos centrales, y como lo plantea Freire: "No puedo negar mi atención decidida y amorosa a la problemática más personal de este o aquel alumno o alumna. [...]. No puedo negar mi condición de persona, de la que se deriva, a causa de mi apertura humana, cierta dimensión terapéutica" (Freire, 2004, p.138). 
Ahora bien, la educación, como cualquier proceso de interacción humana, está mediada por relaciones de poder y por los conflictos que suscitan intereses y perspectivas culturales diferentes (Mejía, Awad, 1999; Bruner, 1998). Esta condición implica asumir una perspectiva plural ante la realidad, "respaldado por la voluntad de negociar nuestras diferencias en la manera de ver el mundo" (Bruner, 1998, p.43).

Desde esta experiencia, las propuestas de Habermas respecto de la construcción de consensos basados en la argumentación y en el establecimientos de criterios aceptados por todos los actores (Habermas, 1987) y los planteamientos de negociación cultural (Mejía, Awad, 1999) son insuficientes para comprender un proceso de educación dialógica como el vivido en el programa. Precisamente porque se reconoce la existencia de conflictos y relaciones de poder en el escenario educativo, habría que tener en cuenta la dimensión hermenéutica del diálogo fundada en la comprensión:

Cuando intentamos entender un texto no nos desplazamos hasta la constitución psíquica del autor, sino que, ya que hablamos de desplazarse, lo hacemos hacia la perspectiva bajo la cual el otro ha ganado su propia opinión. Y esto no quiere decir sino que intentamos que se haga valer el derecho de lo que el otro dice. Cuando intentamos comprenderle hacemos incluso lo posible por reforzar sus propios argumentos. (Gadamer, 2006, p.361)

Este desplazarse no es ni empatía de una individualidad en la otra, ni sumisión del otro bajo los propios patrones; por el contrario, significa siempre un acenso hacia una generalidad superior, que rebasa tanto la particularidad propia como la del otro. El concepto de horizonte se hace aquí interesante porque expresa esa panorámica más amplia que debe alcanzar el que comprende. (Gadamer, 2007, p.375)

\section{Consideraciones finales}

Es necesario tener en consideración que los resultados de la educación para la salud no constituyen un asunto neutral, pues dependerán de las concepciones pedagógicas y de las intenciones de los actores. En este orden de ideas, habrá que tener en cuenta también el sentido que los resultados tienen para los educandos y avanzar hacia la construcción de indicadores como acuerdos entre los educandos y educadores, en el marco de una perspectiva dialógica. Ahora bien, una condición dialógica precisa de una actitud de respeto hacia el otro, de sus conocimientos, sus sentimientos y de su contexto social, económico y cultural. Así mismo una disposición para aprender en el diálogo, para dejarse transformar y para extender su horizonte de sentido en la medida en que puede localizar sus propios puntos de vista dentro de un marco de significado más amplio e incluyente. Esto quiere decir que un horizonte de sentido enriquecido le permite relativizar sus propias concepciones y posiciones hacia la realidad y hacia el otro. De esta manera se podrá avanzar hacia el desarrollo de acciones educativas más productivas y satisfactorias para ambos actores.

\section{Colaboradores}

Los autores Miriam Bastidas, Julio Nicolás Torres, Gloria Matilde Escobar y Fernando Peñaranda trabajaron juntos en todas las etapas de la producción del manuscrito. Las autoras Francy Nelly Pérez y Adriana Arango participaron en el diseño de la investigación, en el trabajo de campo y en el análisis de la información, además revisaron el manuscrito final. 


\section{Referencias}

ACHARD, P. Discurso biológico y orden social: crítica de las teorías biologistas, psicología y ciencias sociales. México: Editorial Nueva Imagen, 1980.

BASTIDAS, M. et al. El diálogo de saberes como posición humana frente al otro: referente ontológico y pedagógico en la educación para la salud. Invest. Educ. Enferm., v.27, n.1, p.104-11, 2009.

BASTIDAS-ACEVEDO, M. et al. La comprensión de los significados que del programa de crecimiento y desarrollo tienen sus actores: un paso hacia su cualificación. Cienc. Saude Colet., v.14, n.5, p.1919-28, 2007.

BOURDIEU, P.; PASSERON, J.C. La reproducción: elementos para una teoría del sistema de enseñanza. 3.ed. México: Distribuciones Fontamara, 1988.

BRUNER, J. Actos de significado: más allá de la revolución cognitiva. Madrid: Alianza Editorial, 1998.

CARVALHO, S. Saúde coletiva e promoção da saúde: sujeito e mudança. São Paulo: Hucitec, 2005.

CENDALES, L. El dialogo una posibilidad de reflexión-acción para los educadores. Educ. Hoy, v.30, n.146, p.71-8, 2001.

COLOMBIA. Ministerio de Salud. Resolución 0412 del 2000, del 25 de febrero: por la cual se establecen las actividades, procedimientos e intervenciones de demanda inducida y obligatorio cumplimiento y se adoptan las normas técnicas y guías de atención para las acciones de protección específica y detección temprana y la atención de enfermedades de interés en salud pública. Bogotá: El Ministerio, 2000.

FALS BORDA, O. Participatory (action) research in social theory: origins and challenges. In: REASON, P.; BRADBURY, H. (Orgs.). Handbook of action research. London: Sage Publications, 2001. p.27-37.

FOUCAULT, M. Historia de la medicalización. Educ. Med. Salud, v.11, n.1, p.3-25, 1977.

FREIRE, P. La educación como práctica de la libertad. México: Siglo XXI, 2005.

Pedagogía de la autonomía: saberes necesarios para la práctica educativa. México: Siglo XXI, 2004.

Política y educación. México: Siglo XXI, 1998.

Pedagogía de la esperanza. México: Siglo XXI, 1996.

Pedagogía del oprimido. México: Siglo XXI, 1975.

GADAMER, H.G. Verdad y método II. Salamanca: Sígueme, 2006.

El estado oculto de la salud. Barcelona: Gedisa, 1996.

GAZZINELLI, M.F. Educação em saúde: conhecimentos, representações sociais e experiências da doença. Cad. Saude Publica, v.27, n.1, p.200-6, 2005.

GONZÁLEZ, E. Corrientes pedagógicas contemporáneas. Medellín: Ed. Universidad de Antioquia, 1999.

GRANDA, E. El saber en salud pública en un ámbito de pérdida del antropocentrismo y ante una visión de equilibrio ecológico. Rev. Fac. Nac. Salud Publica, v.26, n.2, p.65-90, 2008.

GREENE, W.; SIMONS-MORTON, B. Educación para la salud. México: InteramericanaMcGraw Hill, 1988.

HABERMAS, J. Teoría de la acción comunicativa. Madrid: Taurus, 1987. 
HATTON, N.; SMITH, D. Reflection in teacher education: towards definition and implementation. Teach. Teacher Educ., v.11, n.1, p.33-49, 1995.

LINCOLN, Y.; GUBA, E. Paradigmatic controversies, contradictions and emerging confluences. In: DENZIN, N.; LINCOLN, Y.S. (Orgs.). Handbook of qualitative research. 2.ed. Thousand Oaks: Sage Publications Inc., 2000. p.163-88.

MEJÍA, M.; AWAD, M. Educación popular - la negociación cultural: una búsqueda. Bogotá: CINEP, 1999.

MORRELL, C.J. et al. Costs and effectiveness of community postnatal support workers: randomised controlled trial. Br. Med. J., v.321, n.7261, p.593-97, 2000.

NUTBEAM, D. Health literacy as a public health goal: a challenge for contemporary health education and communication strategies into the $21^{\text {st }}$ century. Health Prom. Int., v.15, n.3, p.259-67, 2006.

ORGANIZACIÓN PANAMERICANA DE LA SALUD. ORGANIZACIÓN MUNDIAL DE LA SALUD. Promoción de la salud: logros y enseñanzas extraída entre la carta de Ottawa y la carta de Bangkok y perspectivas para el futuro. Washington: OPS/OMS, 2006.

ORGANIZACIÓN MUNDIAL DE LA SALUD. Carta de Ottawa. Ontario: OMS, 1986.

PANAMERICAN HEALTH ORGANIZATION. Declaration of Alma-Ata. 1978. Disponible en: <http://www.paho.org/english/dd/pin/alma-ata_declaration.htm>. Acceso en: 17 fev. 2011.

PEÑARANDA-CORREA, F. et al. Análisis integral de las prácticas pedagógicas de un programa educativo en Colombia. Salud Publica Méx., v.48, n.3, p.229-35, 2006.

REASON, P.; BRADBURY, H. Introduction: Inquiry and participation in search of a world worthy of human aspiration. In: REASON, P.; BRADBURY, H. (Orgs.). Handbook of action research. Londres: Sage Publications Inc., 2001. p.1-14.

ROBINSON, S.E.; HILL, Y. Our healthier hospital? The challenge for nursing. J. Nurs. Manag., v.7, n.1, p.7-13, 1999.

SALLERAS, L. et al. Educación sanitaria: conceptos y métodos. In: GIL, P. (Org.). Medicina preventiva y salud pública. Barcelona: Elsevier España, 2008. p.265-85.

SEN, A. Capacidad y bienestar. In: NUSSBAUM, M.; SEN, A. (Orgs.). La calidad de vida. México: Fondo de Cultura Económica, 1996. p.54-83.

SERRANO, M.I. Educación para la salud y participación comunitaria. Madrid: Díaz de Santos, 1990.

SHARMA, M.; ROMAS, J. Theoretical foundations of health education and health promotion. Massachusetts: Jones and Bartlett, 2007.

STENHOUSE, L. La investigación como base de la enseñanza. In: RUDDUCK, J.; HOPKINS, D. (Orgs.). La investigación como base de la enseñanza. 5.ed. Madrid: Morata, 2004. p.158-78. (Selección de textos).

TONES, K. Reveille for radicals! The paramount purpose of health education? Health Educ. Res., v.1, n.1, p.1-5, 2002. 
PEÑARANDA-CORREA, F. et al. A práxis como a base de uma educação em saúde alternativa: um estudo de pesquisa-ação no programa de crescimento e desenvolvimento em Medellín, Colômbia. Interface - Comunic., Saude, Educ., v.15, n.39, p.997-1008, out./dez. 2011.

Propondo a reflexão sobre a educação problematizadora, em um ambiente de educação para a saúde, e contribuir para seu desenvolvimento teórico, foi desenvolvida pesquisaação tendo como objeto o programa de crescimento e desenvolvimento em Medellín, Colômbia. O estudo envolveu o uso da observação participante das reuniões do programa de monitorização do crescimento e desenvolvimento de crianças, e a análise da informação focada em um processo reflexivo. Durante o processo, os educadorespesquisadores analisaram suas próprias práticas pedagógicas, visando transformar a educação em saúde numa prática mais dialógica e produtiva. Encontraram-se dificuldades para mudar a educação tradicional no âmbito dos serviços de saúde, mas evidenciou-se a importância da reflexão na transformação da prática. O educando como sujeito, com experiências e condições particulares, contribuiu para a compreensão da educação centrada no ser humano. Ressalta-se a necessidade de se considerarem os adultos como atores centrais e a Educação Infantil como eixo do programa.

Palavras-chave: Educação em saúde. Educação infantil. Crescimento e desenvolvimento. 